\title{
Properties of Galactic Outflows: Measurements of the Feedback from Star Formation
}

\author{
Crystal L. Martin ${ }^{1,2}$
}

\begin{abstract}
Properties of starburst-driven outflows in dwarf galaxies are compared to those in more massive galaxies. Over a factor of $\sim 10$ in galactic rotation speed, supershells are shown to lift warm ionized gas out of the disk at rates up to several times the star formation rate. The amount of mass escaping the galactic potential, in contrast to the disk, does depend on the galactic mass. The temperature of the hottest extended X-ray emission shows little variation around $\sim 10^{6.7} \mathrm{~K}$, and this gas has enough energy to escape from the galaxies with rotation speed less than approximately $130_{-40}^{+20} \mathrm{~km} \mathrm{~s}^{-1}$.
\end{abstract}

Subject headings: galaxies: evolution, formation, ISM

\section{Introduction}

Most models of galaxy formation and evolution contain a critical parameter called feedback. It describes the efficiency with which massive stars reheat the surrounding interstellar medium (ISM) and is thought to have a particularly strong impact on the star formation history of low mass galaxies (Dekel \& Silk 1986, Larson 1974). In CDM-based models for the hierarchical assembly of galaxies, strong differential feedback seems to be required to reproduce the observed galaxy luminosity function and the mass - metallicity relation among galaxies (Kauffman, Guiderdoni, White 1994; Cole et al. 1994; Somerville \& Primack 1998). Given the growing recognition of the importance of feedback and the spatial resolution limits of numerical simulations, empirical descriptions on scales $\gtrsim 1 \mathrm{kpc}$ are needed. Relevant observations of the warm and hot ISM in nearby galaxies are compiled here, and the implications for feedback recipes and galaxy evolution are discussed.

\footnotetext{
${ }^{1}$ Hubble Fellow

${ }^{2}$ Space Telescope Science Institute, 3700 San Martin Drive, Baltimore, MD 21218
} 


\section{Data: Galaxies with Strong Feedback}

A sample of dwarf, spiral, and starburst galaxies was constructed from the literature on galactic winds and extraplanar, diffuse ionized gas (DIG). Most of these galaxies have at least one region where the surface brightness approaches the empirical limit of $L \approx 2.0 \times 10^{11} \mathrm{~L}_{\odot} \mathrm{kpc}^{-2}$ (Meurer et al. 1997). Assuming the slope of the stellar initial mass function (IMF) is Salpeter $(\alpha=2.35)$, the corresponding star formation rate of 1 to $100 \mathrm{M}_{\odot}$ stars is $\dot{\mathrm{M}}_{*} \sim 14 \mathrm{M}_{\odot} \mathrm{yr}^{-1} \mathrm{kpc}^{-2}$. This scale, Leitherer \& Heckman (1995), is used for the star formation rate (SFR) throughout this paper, and extending the IMF to $0.1 \mathrm{M}_{\odot}$ would increase the SFRs by a factor of 2.55 .

\subsection{Dwarf Galaxies}

Large expanding shells of warm ionized gas are common in dwarf galaxies with starburst, i.e. high surface brightness, regions (e.g. Hunter \& Gallagher 1990; Meurer et al. 1992; Marlowe et al. 1995; Hunter \& Gallagher 1997; Martin 1998), but these galaxies are not particularly representative of the dwarf galaxy population. The local number density of dwarf galaxies is sensitive to a survey's surface brightness limit (e.g. Dalcanton 1997) and unknown at the level of a factor of at least 2 to 3. Samples that include some lower surface brightness dwarf irregular galaxies, Hunter, Hawley, \& Gallagher (1993, HHG) for example, have mean $M_{H I} / L_{H \alpha}$ about 1 dex higher than samples of blue amorphous dwarfs (Marlowe et al. 1995). While extraplanar, expanding filaments were found in 7 of 12 galaxies in the latter sample, only 2 of the 15 galaxies with inclinations $i>60^{\circ}$ in the HHG sample even contain extended filaments. Even the HHG sample might be missing more than $1 / 2$ the dwarfs, so the fraction of nearby dwarf galaxies currently in an outflow stage is unlikely to be more than 5\%. Only galaxies with high star formation rates per unit area are discussed in this paper. The frequency of expanding shells is similar to the Marlowe et al. sample, but a broader range of morphological types is included. The absolute magnitude of the galaxies ranges from $M_{B} \approx-13$ to $M_{B}=-18.5$ and $0.84<\left(M_{H I} / L_{H \alpha}\right) /\left(\mathrm{M}_{\odot} / \mathrm{L}_{\odot}\right)<3.17$. Expanding shells were detected in 12 of 14 galaxies using $\mathrm{H} \alpha$ longslit, echelle spectra, and the filaments are clearly extraplanar in 6 galaxies (Martin 1998).

Star formation rates for these dwarf galaxies were derived from the integrated $\mathrm{H} \alpha$ fluxes after correcting for Galactic extinction (Paper I). The intensity of large star forming complexes in many of the dwarfs with strong $\mathrm{H} \alpha$ emission and extraplanar emission reaches several $\mathrm{M}_{\odot} \mathrm{yr}^{-1} \mathrm{kpc}^{-2}$. Averaged over the optical area of a galaxy (i.e. $\pi R_{25}^{2}$ ), however, a typical star formation rate is $1.14 \pm 0.11 \times 10^{-3} \mathrm{M}_{\odot} \mathrm{yr}^{-1} \mathrm{kpc}^{-2}$. Only two galaxies, NGC 1569 and NGC 4449, have secure detections of extended, thermal X-ray 
emission (Della Ceca et al. 1996, 1997). X-ray emission has been detected from several others - NGC 5253, NGC 4214, NGC 1705, IZw18, and VIIZw403, but the thermal emission is not unambiguously resolved from point sources. The peculiar galaxy M82, which is not much more luminous than a dwarf galaxy, also has an X-ray halo (e.g. Strickland et al. 1997).

\subsection{Comparison Sample}

Spiral disks with high areal star formation rates show extraplanar DIG (cf. Table 2 in Rand 1996). The typical spiral galaxy has DIG in the spiral arms, but extraplanar plumes are only present above particularly active sites of localized star formation (Walterbos \& Braun 1994; Wang \& Heckman 1997). This paper examines the DIG in 6 edge-on galaxies with $L_{F I R} / D_{25}^{2}>1 \times 10^{40} \mathrm{ergs} / \mathrm{s} / \mathrm{kpc}^{2}$, or star formation rates greater than $2 \times 10^{-4} \mathrm{M}_{\odot} \mathrm{yr}^{-1} \mathrm{kpc}^{-2}$. The far-infrared luminosity was adopted as the star formation indicator since extinction corrections dominate the $\mathrm{H} \alpha$ luminosity for these galaxies, but the uncertainties in the SFR may be as large as a factor of two (e.g. Sauvage \& Thuan 1992).

Measurements of X-ray halo properties are drawn from Dahlem, Weaver, and Heckman (1998, DWH) who have combined the available ROSAT and ASCA data for a flux limited sample of nearby edge-on starburst galaxies. It is not surprising that some of these galaxies are common to Rand's sample, since filaments protruding from the nucleus account for much of the extended DIG emission. The mean $L_{I R} / D_{25}^{2}$ of the starburst sample, $\sim 2.6 \pm 4.0 \times 10^{-3} \mathrm{M}_{\odot} \mathrm{yr}^{-1} \mathrm{kpc}^{-2}$, is about an order of magnitude higher than that of Rand's sample. More meaningful concentration indices like $L_{I R} / \pi R_{e, H \alpha}^{2}$ give areal star formation rates of order one $\mathrm{M}_{\odot} \mathrm{yr}^{-1} \mathrm{kpc}^{-2}$ but are often not well-defined due to severe extinction (Lehnert \& Heckman 1996). Much (25\% to $78 \%$ ) of the massive star formation in the local universe takes place within the central $\sim 1 \mathrm{kpc}$ of galaxies like these (Gallego et al. 1995; Heckman 1998), and the emission line widths, shock-like line ratios, and $\mathrm{H} \alpha$ morphology demonstrate that minor-axis outflows are prevalent (Lehnert \& Heckman 1995).

\section{Results}




\subsection{Disk Mass Loss Rates in Dwarf Galaxies}

Prominent shells and filaments are plainly visible in the $\mathrm{H} \alpha$ imagery of the star-forming dwarf galaxy sample. The subset of filaments which comprise large, expanding shells were kinematically identified in Paper I, and their $\mathrm{H} \alpha$ luminosities are tabulated in Table 3 of Paper I. The density in the extended filaments $n_{e}$, is too low to measure with common line ratio diagnostics (e.g. Osterbrock 1989), so shell masses were parameterized in terms of an unknown volume filling factor $\epsilon$, where $n_{r m s}^{2}=\epsilon n_{e}^{2}$. For any measured luminosity, condensations along the sightline reduce the inferred mass, $M \propto \epsilon^{1 / 2}$, but increase the inferred pressure, $P \propto \epsilon^{-1 / 2}$. As illustrated in Figure \&, varying the volume filling factor, $\epsilon$, from 1 (upper left) to $10^{-5}$ (lower right) changes the inferred mass of the largest shells from several times $10^{6} \mathrm{M}_{\odot}$ to $10^{4} \mathrm{M}_{\odot}$. The pressure in the warm filaments is unlikely, however, to exceed the pressure of the hot gas which presumably fills the interior cavity.

For one of the nearest starburst galaxies, NGC 1569, extended, soft X-ray emission was identified in ROSAT hardness maps, and a soft, thermal component was required to fit the integrated ASCA spectrum (Della Ceca et al. 1996). The thermal pressure derived from this model depends more on the choice of plasma model and shell volume than any calibration uncertainties, and the acceptable range is illustrated in Figure 1 by vertical lines. The Meka thermal model with the large, H95 volume gives the lowest $P_{x}$, while the $\mathrm{H} \alpha$ shell volume plus Raymond-Smith spectral fit allows a pressure roughly 4 times higher. Pressure equilibrium between the hot and warm gas requires $-3<\log \epsilon<-2$ for each of the large shells protruding from NGC 1569.

The same argument implies $\log \epsilon \approx-2$ for the large $\mathrm{H} \alpha$ shells associated with the extended X-ray emission from NGC 4449. Figure 1 $1 \mathrm{~b}$ illustrates the substantial range of pressure allowed by the volume estimates. The pressure of the very soft thermal component $(0.24 \mathrm{keV})$ is similar to, actually $1 / 2$ as large as, that of the soft component $(0.82 \mathrm{keV})$. For M 82, Figure 1 $\mathrm{c}$, filling factors from $10^{-4}$ to $10^{-2}$ would bring the pressure of the warm ionized filaments into the $P_{x}$ range measured along the outflow (Strickland et al. 1997). The filling factor seems to be about 10 times lower in the M 82 outflow than in NGC 1569 and NGC 4449, and a similar difference has been measured for their HII region filling factor (Martin 1997, Table 6). In contrast, the very high filling factor inferred for NGC 4449 from the DGH97 volume estimate would be difficult to reconcile with the very low HII region ionization parameter.

The gas pressures in Figure 1 are high compared to the local Milky Way ISM but quite reasonable. Adiabatic bubble models for the shells' expansion predict pressures of $2.0 \pm 1.4 \times 10^{5} k \mathrm{~K} \mathrm{~cm} \mathrm{~cm}^{-3}, 1.3 \pm 2.2 \times 10^{5} \mathrm{k} \mathrm{K} \mathrm{cm}^{-3}$, and $7.2 \pm 1.4 \times 10^{5} k \mathrm{~K} \mathrm{~cm}^{-3}$ in NGC 1569, NGC 4449, and M 82 respectively. (These values assume a mean ambient 
density of $n_{0} \approx 0.1 \mathrm{~cm}^{-3}$, use the power and ages from Table 2 of Paper I, and assign $5 / 11$ of the energy to the hot, shocked gas (Koo \& McKee 1992).) The magnetic pressure, $B^{2} / 8 \pi$, is 1 to 2 orders of magnitude smaller than this in NGC $1569,4.2 \times 10^{4} \mathrm{k} \mathrm{K} \mathrm{cm}^{-3}$ (Israel \& deBruyn 1988), and in M 82, $3.5 \times 10^{3} \mathrm{k} \mathrm{K} \mathrm{cm}^{-3}$ (Seaquist \& Odegard 1991). The filling factors derived from the pressure equilibrium argument are therefore expected to be accurate to better than a factor of 10. Based on these results, the warm ionized gas masses in Tables 3 and 5 of Paper I would be more revealing if parameterized in terms of $\epsilon=0.01$ rather than the original $\epsilon=0.1$. The corrections to the mass obtained using the $\epsilon$ values derived above for NGC 1569, NGC 4449, and M 82 are then minor. For a particular galaxy, a lower limit on the disk mass loss rate is simply the sum of its shell masses divided by the age of the oldest shell,

\subsection{Reheating Efficiency}

Figure 2 shows the ratio of disk mass loss rate, $\dot{M}_{w}$, to star formation rate as a function of circular velocity. Although the warm ionized shells typically contain at most a few percent of the galactic gas mass, they lift gas out of the disk at rates comparable to the rate gas goes into new stars. No trend is seen with $V_{c}$ over the luminosity/mass interval of the dwarf sample - the solid symbols.

Comparison of the reheating efficiency measured in the dwarf galaxies to that in more massive disk galaxies is not straightforward. The mass of the extended DIG in the spirals NGC 4013, NGC 4302 and NGC 3079 was computed from emission profiles $n_{r m s}^{2}(R, z)=<n_{r m s}^{2}>_{0} e^{-z / z_{0}}$ for $R \leq R_{0}$ - fit to deep H $\alpha$ images (Rand 1996; Veilleux et al. 1995). The halo DIG mass for NGC 891 is from Dettmar (1990). For NGC 4631, the hot gas mass loss rate, $\dot{M}_{x}$, from Wang et al. (1995) was substituted for $\dot{M}_{w i m}$. All measurements were scaled to a common filling factor of $\epsilon=10^{-2}$ for comparison to the dwarf galaxies sample, but measurements of the latter were not corrected for [NII] emission in the filter bandpass. The gas dynamical timescale was set equal to the emission measure scale height divided by the sound speed at $10^{4} \mathrm{~K}$. Open symbols in Figure 2 show the resulting ratio, $\dot{M}_{w} / \dot{M}_{*}$, for these five galaxies. Any point in this diagram is uncertain by a factor of 2-3, but it is remarkable that - over a factor of nearly 10 in galactic rotation speed - the upper envelope shows little variation around $\dot{M} / \dot{M}_{*} \sim 5$. This upper limit probably indicates something fundamental about the reheating efficiency. In particular, it is more related to the areal density of stars than the depth of the potential. 


\subsection{Galactic Mass Loss}

The fate of the gas in the expanding shells depends on the gravitational potential of the galaxy. For a measured rotation speed (Paper I), the distribution of matter in both the galactic disk and the halo affect the estimated depth. For example, the escape velocity at $R\left(\max V_{c}\right)$ is at least $1.414 V_{c}$ but increases to $3.55 V_{c}$ or $2.57 V_{c}$ for spherical, isothermal halos extending, respectively, to 100 times or 10 times this radius. The shells in NGC 1569, one shell in Sextans A, and one shell NGC 3077 have projected expansion speeds greater than $1.414 V_{c}$; but only one of the shells in NGC 1569 is expanding faster than $3.55 V_{c}$. Hence, even in dwarf galaxies, much of the warm, ionized gas blown out of a disk probably remains bound to the galaxy.

The fate of the hot gas confined by the shells may be different. Supershells accelerate when they reach several gas scale heights and break up from Rayleigh-Taylor instabilities (MacLow, McCray, \& Norman 1989). The hot, interior gas exits at the sound speed. In the absence of radiative losses, gas hotter than $T_{\text {esc }}=1.5 \times 10^{5}\left(v_{\text {esc }} / 100 \mathrm{~km} / \mathrm{s}\right)^{2}$ escapes the galactic potential. This critical temperature represents a specific enthalpy equal to $1 / 2 v_{\text {esc }}^{2}$. Figure 3 shows its variation with galactic rotation speed for the three $v_{e s c} / V_{c}$ ratios discussed above. The temperature of the hot gas in NGC 1569 and NGC 4449 is well above all these limits. The temperature of the M 82 outflow also exceeds the escape temperature if the halo is severely truncated - i.e. the bold line (see Sofue et al. 1992). Solar metallicity gas at $T=10^{6.8} \mathrm{~K}$ and $n=0.01 \mathrm{~cm}^{-3}$ cools radiatively in $\sim 2 \times 10^{8} \mathrm{yr}$ (Sutherland \& Dopita 1993), and the halo gas could reach a radius of $\sim 40 \mathrm{kpc}$ in this time. The mass of the hot outflow in NGC 1569 is $M_{x}=6.12-6.99 \times 10^{5} \mathrm{M}_{\odot} \sqrt{V / 1 \mathrm{kpc}^{3}}$, or $6.3-7.2 \times 10^{5} \mathrm{M}_{\odot}$; and the soft and very soft components in NGC 4449 contain $M_{x}=5.3 \times 10^{5} \mathrm{M}_{\odot}$ and $M_{x}=7.9 \times 10^{5} \sqrt{V / 1 \mathrm{kpc}^{3}} \approx 8.9 \times 10^{5} \mathrm{M}_{\odot}$ respectively. The X-ray emitting gas contains about as much mass as the $\mathrm{H} \alpha$ shells, so the disk mass loss rates in Figure 2 are indicative of the galactic mass loss rate as well.

The importance of this result for modeling feedback is amplified by measurements of $T_{x}$ in more massive galaxies. The temperature constraints found for NGC 891 (Bregman \& Houck 1997) and NGC 4631 (Wang et al. 1995) are shown in Figure 3 along with the sample re-analyzed by Dahlem et al. (1998). The temperature of the hot gas in these galaxies is similar to that in the two dwarf galaxies and M82, about $T_{x} \sim 10^{6.8} \mathrm{~K}$. Although foreground Galactic absorption could hide a lower temperature thermal component in several of these galaxies, the ASCA spectra which extend to $10 \mathrm{keV}$ would have detected a hotter thermal component if it were present. Since the minimum in the cooling curve occurs at a higher temperature, $T \approx 10^{7.4} \mathrm{~K}$, the temperature uniformity must reflect the reheating efficiency of massive stars. As illustrated in Figure 3, the escape temperature from an extended halo 
rises above the hot gas temperature at a circular velocity $\sim 130 \mathrm{~km} \mathrm{~s}^{-1}$. The hot gas in the outflow is therefore expected to form a bound halo around larger galaxies.

\section{Discussion: Recipes and Implications}

To describe the global impact of star formation on the ISM, a very simple empirical feedback recipe is proposed. Three components of interstellar gas, which can be referred to as cold, warm, and hot must be identified. Use the Schmidt law parameterization of Kennicutt (1998) to estimate the global SFR. If the SFR averaged over the area of the stellar disk exceeds a few times $10^{-4} \mathrm{M}_{\odot} \mathrm{yr}^{-1} \mathrm{kpc}^{-2}$, then transfer warm $\left(\sim 10^{4} \mathrm{~K}\right)$ disk gas to the halo at a rate of a few times the star formation rate. Generate hot, $\sim 10^{6.7} \mathrm{~K}$, gas at a similar rate and remove it from the halo if the rotation speed is less than $\sim 130_{-40}^{+20} \mathrm{~km} \mathrm{~s}^{-1}$.

Comparison of this empirical recipe to those in the semi-analytic galaxy formation models (SAMs) of the Munich, Durham, and Santa Cruz groups provides some insight into the impact of such a recipe. The observations indicate that the differential aspect of the feedback is the escape fraction of hot gas from the halo. For simplicity, the empirical recipe presents a sharp transition from ejection to retention, but a milder increase in ejection efficiency toward lower circular velocity could still be quite reasonable. The disk reheating rate was found to be insensitive to $V_{c}$ in contrast to the common prescription in the SAMs

where $\dot{M}_{\text {reheat }} / \dot{\mathrm{M}}_{*} \propto V_{c}^{\alpha}$ with $\alpha \sim-2$. All three groups enhance the reheating efficiency in the dwarfs, but the temperature assigned to this reheated gas, or equivalently its fate, differ. If the reheated gas is ejected from the halo (e.g. Cole et al. 1994; SP98), the prescription becomes very similar to the empirical recipe. These ejection models flatten the faint-end of the luminosity function more than models which retain the reheated gas (e.g. KGW94). The empirical feedback recipe will not, however, suppress star formation in small halos as strongly as the Durham prescription. The latter lowers the star formation effeciency in dwarfs in addition to increasing the feedback, and Figure 7 of SP98 indicates this causes too much curvature at the faint end of the Tully-Fisher relation. The mass - metallicity relation depends on assumptions about the composition of outflow but would seem to be in a reasonable regime (e.g. Figure 13 of SP98).

The outflows observed in nearby dwarf galaxies do not expel the entire disk over the lifetime of individual starburst regions. For example, the wind in NGC 1569 might expel $\sim 0.3 \mathrm{M}_{\odot} \mathrm{yr}^{-1}$ over $10^{8} \mathrm{yr}$, or $3 \times 10^{7} \mathrm{M}_{\odot}$ of the disk. If this mass is swept out of the central cylinder of radius $500 \mathrm{pc}$ and height $1 \mathrm{kpc}$, the concentration of the ejected disk material is $M_{d} / r_{d}=0.07 V_{30}^{-2}$ in units of the halo mass to scalelength $M_{h} / a_{h}$. Much larger concentrations, like $M_{d} / r_{d} \sim 1-20$, must be ejected to unbind a substantial amount of 
the central cusp in the dark matter distribution (Navarro, Eke, \& Frenk 1996). The mass lost from local star-forming dwarfs does not seem to be sufficient to generate the dark matter cores observed in some low surface brightness dwarf galaxies. If areal SFRs were $\gtrsim 10$ times higher in halos of a given circular velocity at high redshift, then the fraction of warm and cold gas escaping the halo would have been more significant. This fraction would also be increased if environmental effects both trigger starbursts and truncate their surrounding dark matter halos (e.g. M82, Sofue et al. 1992). It is unclear, however, whether the reheating could have smoothed out the gas distribution enough to prevent the severe angular momentum losses that plague current N-body/gasdynamical simulations of galaxy formation (Navarro \& Steinmetz 1997).

The empirical recipe can be improved with further work. Only the most vigorously star-forming local galaxies were considered in this paper, but the critical areal SFR for supershell blowout and its sensitivity to $\mathrm{HI}$ scale height could be measured. The total mass at temperatures, $\sim 10^{5}-10^{6} \mathrm{~K}$, needs to be better constrained. It is similar to that in the hot $10^{6.7} \mathrm{~K}$ phase for NGC 4449 and NGC 4631 - two galaxies with foreground absorption low enough to allow detection in the X-ray spectrum. Both molecular and neutral atomic gas have been detected in galactic outflows (Sofue et al. 1992; Toshihiro et al. 1992; Heckman \& Leitherer 1997), but the ubiquity of a cold component and its mass need to be determined. The biggest systematic uncertainty affecting the galactic mass loss rates is radiative losses. Mass loaded outflows could radiate more of the thermal energy reservoir than assumed here, so a better understanding of the transfer of mass and energy between different phases of gas in the outflows is needed. Feedback can be countered to some degree by adjusting cosmological parameters, particularly the slope of the power spectrum on small scales (SP98). Tighter empirical constraints on the feedback would help ensure SAMs narrive at the physical solution.

Support for this work was provided by NASA through Hubble Fellowship grant \#HF-01083.01-96A awarded by the Space Telescope Science Institute, which is operated by the Association of Universities for Research in Astronomy, Inc., for NASA under contract NAS 5-26555. This work benefited from discussions with Carlos Frenk, Tim Heckman, Guinevere Kaufmann, Cedric Lacey, Julio Navarro, Rachel Somerville, and Matthias Steinmetz at the Aspen Center for physics. Literature searches were performed with NED, the NASA/IPAC Extragalactic Database, a facility operated by the Jet Propulsion Laboratory, Caltech, under contract with NASA. 


\section{REFERENCES}

Bregman, J. N. \& Houck, J. C. 1997, ApJ, 485, 159.

Cole, S., Aragon-Salamanca, A., Frenk, C. S., Navarro, J. F., \& Zepf, S. E. 1994, MNRAS, $271,781$.

Dahlem, M., Weaver, K. A., \& Heckman, T. M. 1998, preprint.

Dalcanton, J. J. et al. 1997, AJ, 114, 635.

Dekel, A., \& Silk, J. 1986, ApJ, 303, 39.

Della Ceca, R., Griffiths, R. E., Heckman, T. M., \& Mackenty, J. W. 1996, ApJ, 469, 662 (DGHM96).

Della Ceca, R. et al. 1997, ApJ, 485, 581 (DGH97).

Dettmar, R. J. 1992, in Fundamentals of Cosmic Physics, Vol 15, (Gordon and Breach Science Publishers: USA).

Gallego, J., Zamorano, J., Aragon-Salamanca, A., \& Gego, M. 1995, ApJ, 455, 1.

Heckman, T. M. 1998, in Origins, ed. C. Woodward \& J. M. Shull, PASP, ?.

Heckman, T. M., Dahlem, M., Lehnert, M. D., Fabbiano, G., Gilmore, D., \& Waller, W. H. 1995, ApJ, 448, 98.

Heckman, T. M., \& Leitherer, C. 1997, AJ, 114, 69.

Hunter, D. A., \& Gallagher, J. S. III 1997, ApJ, 475, 65.

Hunter, D. A., \& Gallagher, J. S. III 1990, ApJ, 362, 480.

Hunter, D. A., Hawley, W. N., \& Gallagher, J. S. 1993, AJ, 106, 1797.

Israel, F. P., \& de Bruyn, A. G. 1988, A\&A, 198, 109.

Kauffman, G., Guiderdoni, B., \& White 1994, MNRAS, 267, 981.

Kennicutt, R. C. 1998, ApJ, 498, 541.

Koo, B.-C. \& McKee, C. F. 1992, ApJ, 388, 103.

Larson, R. B. 1974, MNRAS, 169, 229.

Leitherer, C., \& Heckman, T. M. 1995, ApJS, 96, 9L.

Lehnert, M. D., \& Heckman, T. M. 1996, ApJ, 472, 546 (LH96).

MacLow, M.-M., McCray, R., \& Norman, M. L. 1989, ApJ, 337, 141.

Marlowe, A. T., Heckman, T. M., Wyse, R. F. G., \& Schommer, R. 1995, ApJ, 438, 563.

Marlowe, A. T., Meurer, G. R., Heckman, T. M., \& Schommer, R. 1997, ApJS, 112, 285. 
Martin, C. L. 1998, ApJ, in press (Paper I).

Martin, C. L. 1998, ApJ, 491, 561.

Meurer, G. R. et al. 1997, AJ, 114, 54.

Meurer, G. R., Freeman, K. C., Dopita, M. A., \& Cacciari, C. 1992, AJ, 103, 60.

Navarro, J. F., Eke, V. R., \& Frenk, C. S. 1996, MNRAS, 283, 72

Navarro, J. F., \& Steinmetz, M. 1997, ApJ, 478, 13.

Osterbrock, D. E. 1989, Astrophysics of Gaseous Nebulae and Active Galactic Nuclei (University Science Books: Mill Valley, CA).

Rand, R. J. 1996, ApJ, 462, 712.

Sofue, Y., Reuter, H.-P., Krause, M. Wielebinski, R., \& Nakai, N. 1992, ApJ, 395, 126.

Seaquist, E. R., \& Odegard, N. 1991, ApJ, 369, 320.

Somerville, R. S., \& Primack, J. R. 1998, MNRAS, submitted, astro-ph/9802268.

Strickland, D. K., Ponman, T. J., \& Stevens, I. R. 1997 A\&A, 320, 378.

Sutherland, R. S. \& Dopita, M. A. 1993, ApJS, 88, 253.

Toshihiro, H. Sofue, Y., Ikeuchi, S., Kawabe, R., Ishizuki, S. 1992, PASJ, 44, L227.

Veilleux, S., Gerald, C., \& Bland-Hawthorn, J. 1995, ApJ, 445, 152.

Walterbos, R. A. M., \& Braun, R. 1994, ApJ, 431, 156.

Wang, J., Heckman, T. M., \& Lehnert, M. D. 1997, ApJ, 491, 114.

Wang, Q. D. et al. 1995, ApJ, 439, 176. 
Fig. 1.-

Mass and pressure of the warm, ionized shells in 3 actively star-forming galaxies. The diagonal lines illustrate the effect of varying the volume filling factor $\epsilon$ from unity (upper left) to $10^{-5}$ (lower right) in increments of 1 dex. Vertical lines denote the pressure of the hot bubbles. Allowed range given by: (a) Mewe and Raymond-Smith models (DGHM96) with volumes from $1.061 \mathrm{kpc}^{3}$ (Paper I) to $4.188 \mathrm{kpc}^{3}$ (Heckman et al. 1995), (b) Meka and Raymond-Smith models (DGH97) with volumes from $1.290 \mathrm{kpc}^{3}$ (Paper I) to $9.4 \mathrm{kpc}^{3}$ (DGH97 scaled to d = 3.6 Mpc). (c) Gradient along outflow (Strickland et al. 1997).

Fig. 2.- Mass ejection efficiency versus maximum HI rotation speed. Filled circles represent the ratio of the WIM mass loss rate, which scales as $\sqrt{\epsilon / 0.01}$, to the galactic SFR in a sample of high SFR dwarf galaxies. Arrows illustrate corrections to the filling factor derived from pressure equilibrium arguments. Open symbols show an analogous ratio for spiral galaxy disks with high areal star formation rates. Pairs of triangles denote upper and lower limits for a galaxy.

Fig. 3.-

Temperature of extended, thermal X-ray emission versus maximum HI rotation speed. Solid symbols denote a second thermal component when detected. Shown are NGC 1569 (Della Ceca et al. 1996, $\square$ ), NGC 4449 (Della Ceca et al. 1997, $\square$ ), M 82 (Strickland et al. 1997, $\triangle$; DHW, ○), NGC 4631 (Wang et al. 1995, $\triangle$ ) NGC 891 (Bregman \& Houck 1997, $\triangle$ ), NGC 253, NGC 3079, NGC 3628, and NGC 2146 (DWH, $\bigcirc$ ). The solid line illustrates the minimum escape temperature - i.e. all the mass is interior to the location where the rotation speed was measured. The dotted lines show the escape temperature from isothermal halos truncated at 10 times and 100 times this radius. 


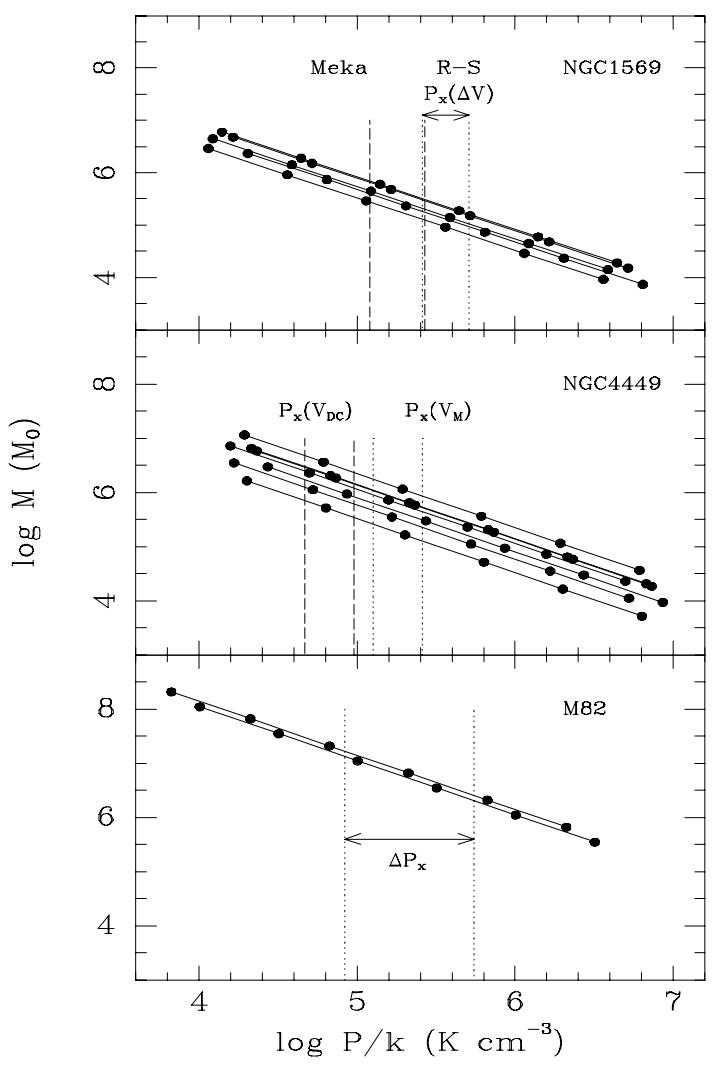




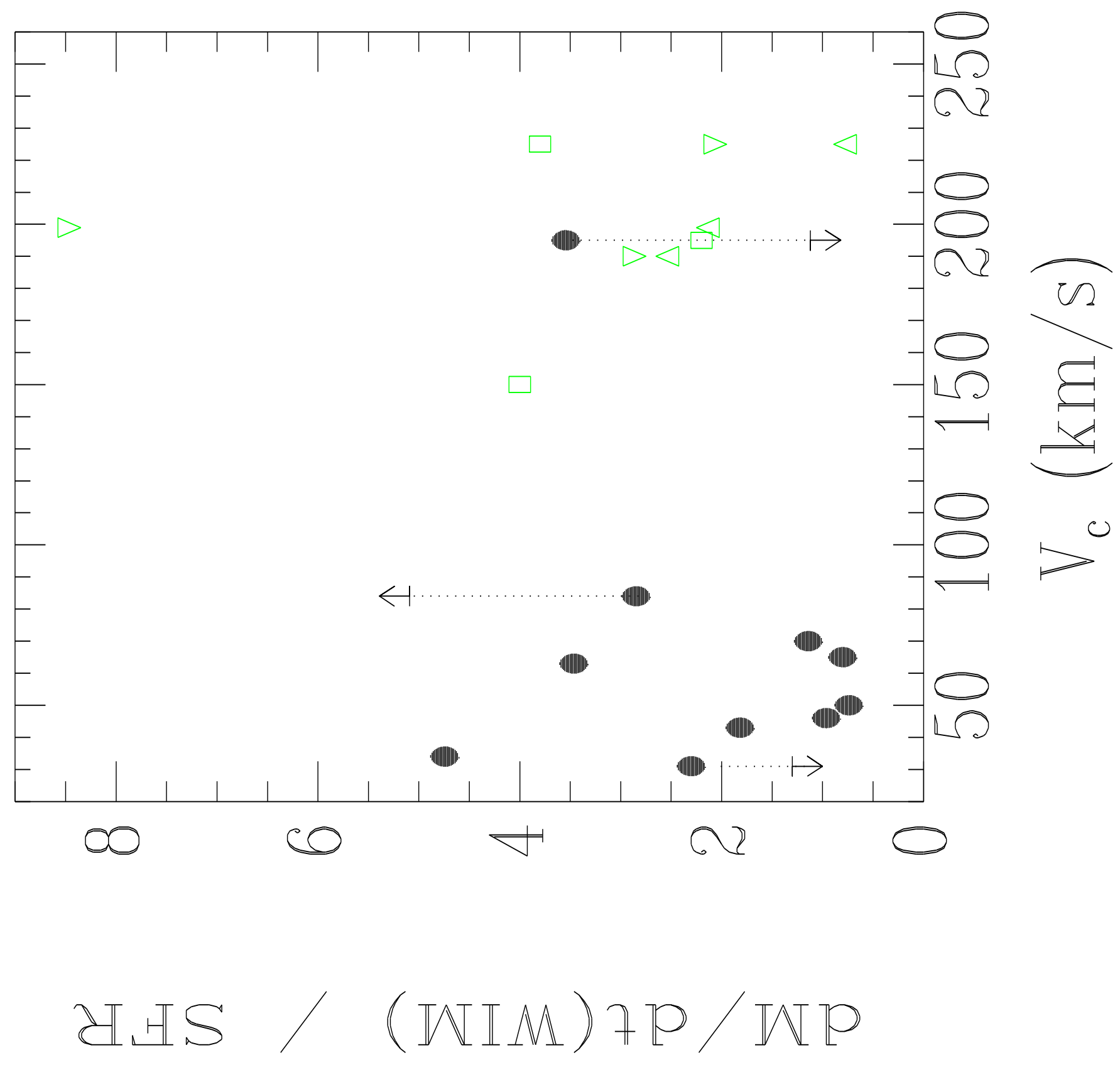




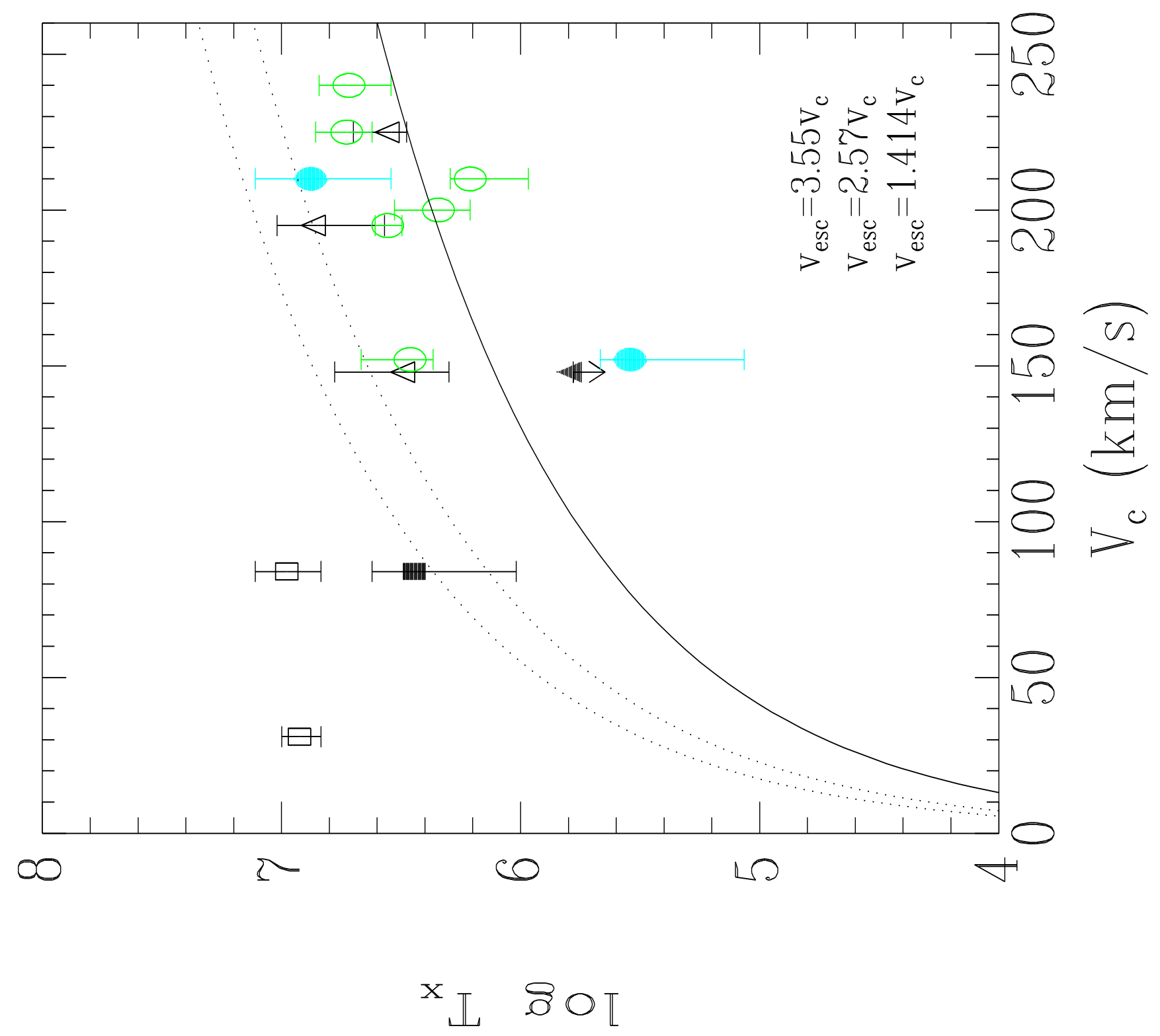

